\title{
Outcomes of prolotherapy for intra-tendinous Achilles tears: a case series
}

\author{
Otto Chan ${ }^{1}$ \\ Ben Havard ${ }^{2}$ \\ Sarah Morton² \\ Mel Pritchard 1 \\ Nicola Maffulli ${ }^{1}$ \\ Tom Crisp ${ }^{1,2}$ \\ Nat Padhiar ${ }^{1,2}$ \\ Jeremy David Perry ${ }^{1,2}$ \\ John King ${ }^{1,2}$ \\ Dylan Morrissey 1,2,3 \\ 1 BMI London Independent Hospital, Stepney Green, \\ London, UK \\ 2 Centre for Sports and Exercise Medicine, \\ William Harvey Research Institute, \\ Queen Mary University of London, \\ Mile End Hospital, London, UK \\ ${ }^{3}$ Bart's Health NHS Trust, London, UK
}

Corresponding author:

Dylan Morrissey

Centre for Sports and Exercise Medicine,

William Harvey Research Institute,

Queen Mary University of London,

Mile End Hospital, Bancroft Road, E1 4DG

London, UK

E-mail:d.morrissey@qmul.ac.uk

\section{Summary}

Background: The intra-tendinous tear is a new pathology that is defined as a discontinuity of fibres situated entirely within the tendon. Prolotherapy involves injecting an irritant, such as hyperosmolar dextrose, to stimulate a tissue healing response and ultimately reduce pain.

Methods: 43 consecutive patients diagnosed with an intra-tendinous tear were included (27 males: 16 females, mean (SD) age 41 (11.3). Patients were injected with $0.4 \mathrm{ml}-1.5 \mathrm{ml}$ (mean $0.8 \mathrm{ml}$ ) of $50 \%$ dextrose and $0.5 \%$ marcaine mixed in a 1:1 ratio. A 4-6 week period of walking boot immobilisation was followed by progressive rehabilitation (6-8 weeks). Outcomes were assessed with a VISA-A questionnaire at baseline, 3 months and a mean 12.6 (7.0) months post-treatment. Ultrasound scans were conducted before treatment and 5.2 (2.3) weeks later to assess sonographic changes.
Results: 30 patients (70\%) responded with VISA-A scores increasing by 31 (30.5) points after 3 months $(f=0.62, p<0.05)$ and by $40(29.3)$ points after 12.60 (7.0) months $(f=0.87, p<0.05)$. After 5.2 (2.3) weeks, echogenicity was significantly reduced $(p<0.05)$ and $27 \%$ of tears were no longer detectable. No significant differences were observed in remaining tears with respect to tear size, tendon thickness or neovascularisation.

Conclusion: Treatment resulted in clinically significant improvements and controlled trials are warranted.

Level of evidence: IV.

KEY WORDS: prolotherapy, Achilles tendon, Achilles tendinopathy, intra-tendinous tear, ultrasound, dextrose.

\section{Introduction}

The Achilles tendon is the largest and strongest tendon in the human body but Achilles injuries are one of the most common foot and ankle problems associated with sport ${ }^{1}$. They are more common in active individuals who place considerable load on the tendon during running and jumping ${ }^{2}$. Complete or partial ruptures, retrocalcaneal bursitis, Sever's disease, plantaris pathologies and tendinopathy at the mid-portion or insertion are well reported in the literature ${ }^{3-5}$. However, a recent study has recognized a new pathology that should be considered ${ }^{9}$.

The intra-tendinous Achilles tear (ITT) is defined as a discontinuity of fibres situated entirely within the tendon (Fig. 1). US imaging typically reveals a thickened tendon with a central hypoechoic area and increased neovascularisation on power Doppler. In contrast to diffuse tendinopathic stiffness, ITT patients present with localised pain that can be reproduced on very localized palpation. Patients are often able to train but cannot reach maximal loading and typically report intense pain when trying to push off at the start of a sprint 6,7 .

Prolotherapy involves injecting an irritant, such as hyperosmolar dextrose, into a joint space, ligament or tendon with the ultimate goal of alleviating pain ${ }^{8}$. The mechanism is not fully understood but there is evidence that an inflammatory response causes growth factor release, which stimulates collagen deposition and tissue repair ${ }^{9}$. There is good evidence that prolotherapy is an effective tendinopathy treatment, that significantly reduces neovascularisation which often correlates with clinical improvement ${ }^{10-13}$. However, 


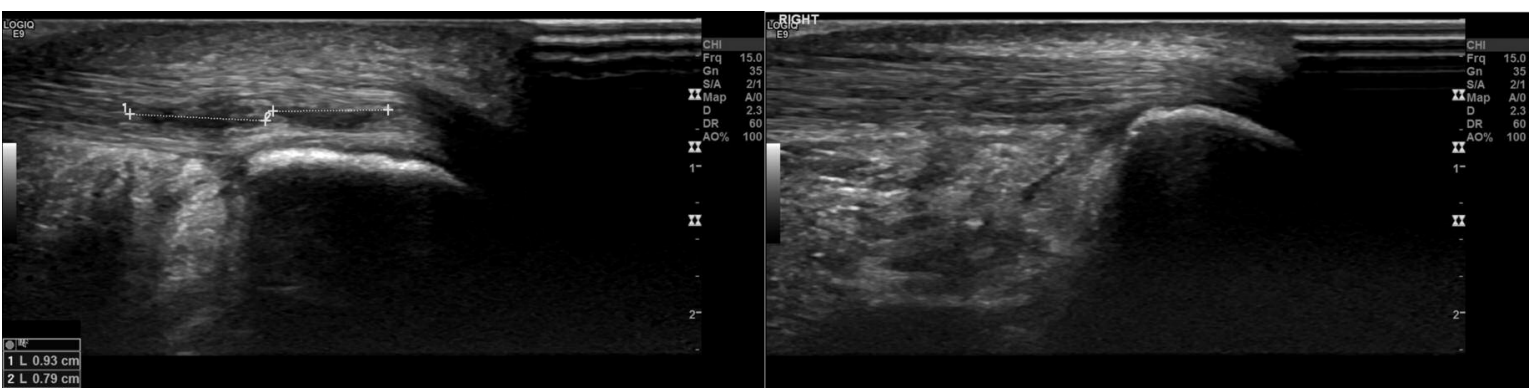

Figure 1. Tendon with two separate intra-tendinous tears before (left) and 6 weeks after prolotherapy treatment (right).

the additional effects on tissue repair suggest that prolotherapy may demonstrate superior results in treating intra-tendinous tears where discrete fibre disruption has occurred ${ }^{14}$. Previous studies have assessed prolotherapy injections in isolation whereas this study aimed to evaluate the effectiveness of a single prolotherapy injection combined with a specific rehabilitation program ${ }^{13}$.

We aimed to provide clinical decision making guidance and explore the effectiveness of an innovative treatment package for ITTs. Objectives included evaluating the effects of dextrose prolotherapy, followed by a period of immobilisation and rehabilitation, on pain and function in patients with an ITT. We hypothesised that pain and function would be significantly improved while follow-up US scans would reveal fewer sonographic abnormalities.

\section{Materials and methods}

This study was designed in accordance with the scientific and ethical principles outlined in Muscles, Ligaments and Tendons Journal to enable an accurate evaluation of the main findings ${ }^{15}$.

\section{Participants}

Patients were included in the study if they were aged 18-65 and had received prolotherapy treatment for an ITT between March 2012 and March 2014. The diagnosis of an ITT was made both clinically on the basis of localised pain on palpation with a negative Simmonds' test, and radiologically, based on the presence of a clearly visualized echopoor area situated entirely within the tendon ${ }^{6}$. All patients received a single injection and were excluded if they had received previous intra-tendinous treatment. A total of 43 patients satisfied the above inclusion criteria. There were 27 males and 16 females, with a mean age of 41 (range 22-64).

\section{Patient reported outcome measures}

These patients were fully informed about the study and were sent a study-specific questionnaire. This in- corporated 3 VISA-A questionnaires as well as some questions relating to the rehabilitation phase. The first two VISA-A questionnaires were completed retrospectively to assess how pain and function had changed from before treatment to 3 months after treatment. The final questionnaire evaluated current symptoms. Ethical approval was obtained from Queen Mary, University of London Research Committee and return of the questionnaire explicitly indicated consent.

The VISA-A questionnaire is a valid and reliable measure of Achilles tendinopathy symptoms but it has been used to evaluate other Achilles pathologies ${ }^{16,17}$. The ITT does not have a specific assessment tool and the VISA-A was felt to be the most suitable substitute. Score increases of 10,12 , and 25 have been regarded as clinically meaningful differences but the precise value has never been formally tested ${ }^{18-21}$. For the purpose of this study we regarded an improvement of 15 points as clinically significant.

\section{Ultrasound examination}

The same experienced MSK consultant radiologist (OC) performed all US examinations and treatments with the same US scanner (Elegra, Siemens, Erlangen Germany). Subjects were positioned in a long-sitting position with their hips flexed and externally rotated, knees flexed and ankles in a neutral position. A $13 \mathrm{MHz}$ transducer with power Doppler imaging was used to examine both Achilles tendons in the longitudinal and transverse planes. Static and video images were obtained to record tear length, tear width and the anteroposterior diameter of the tendon at its thickest point.

The Modified Ohberg scale was used to grade neovascularisation between zero (no Doppler signal) and five (over $90 \%$ of the pathological area contained Doppler signal)22. Echogenicity was assessed in two ways. An ordinal scale from a previous study was used to grade echogenicity between 0 and $3^{10}$. ImageJ software was used to determine the mean grayscale value (brightness) of the tear area (Fig. 2). This was then divided by the mean grayscale value of the tear area and surrounding area (Fig. 3) to yield the relative grayscale value as a representation of the echotexture of the tear relative to the surrounding 

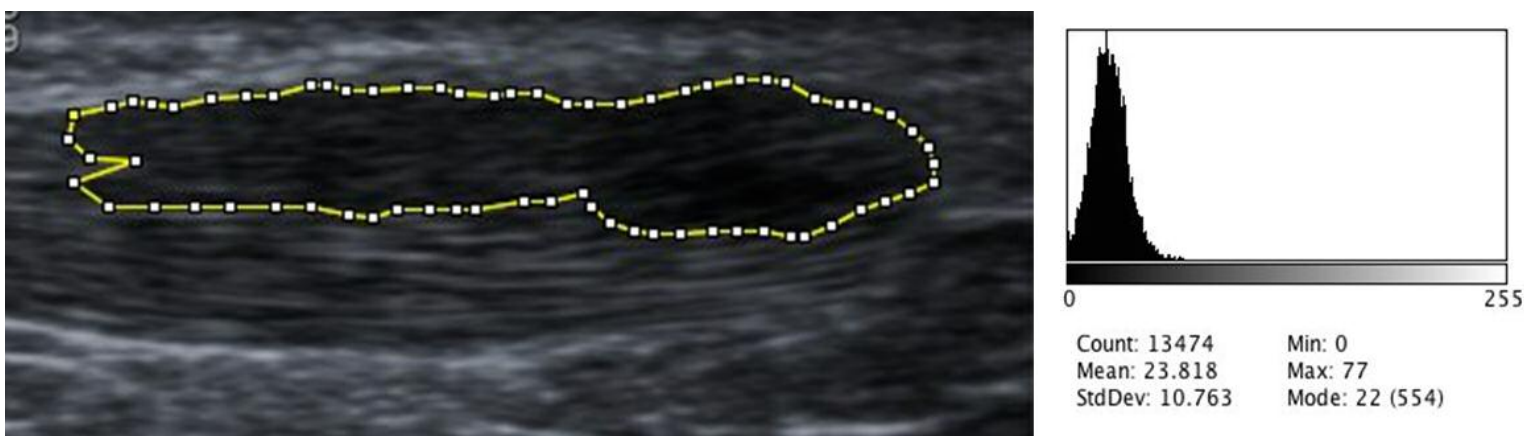

Figure 2. Measurement of tear area only with corresponding histogram of grayscale values.
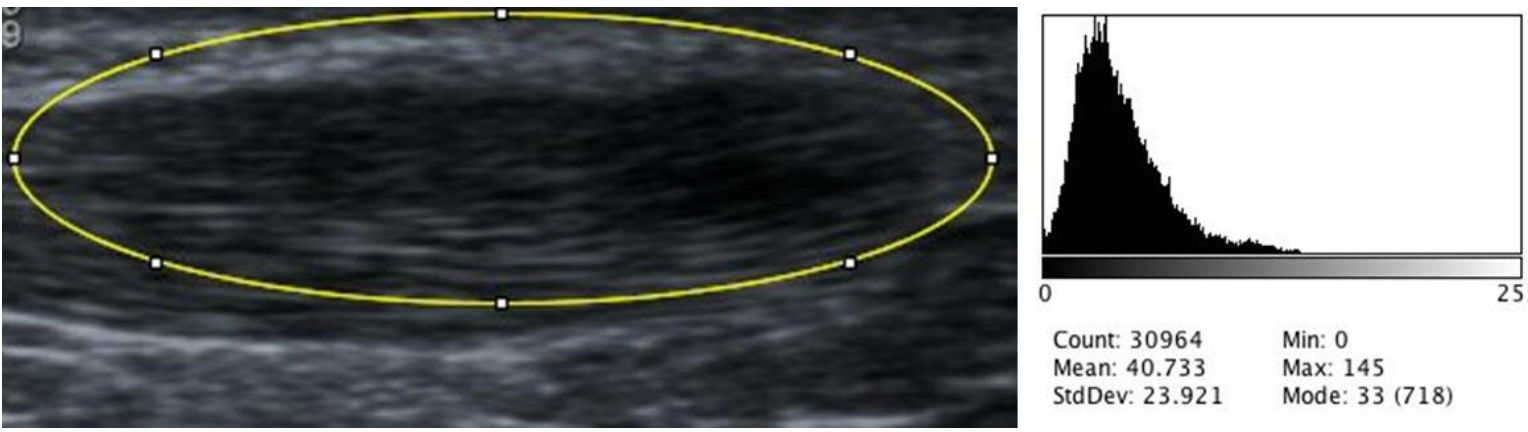

Count: 30964

Mean: 40.733

Min: 0

StdDev: 23.921

Max: 145

Mode: 33 (718)

Figure 3. Measurement of tear and surrounding area with corresponding histogram of grayscale values.

tendon (a value of one would indicate homogeneity). US images were collected before treatment and a mean of 5.2 (SD 2.3) weeks after injection for objective assessment of tear size, grade of neovascularisation, echogenicity and anteroposterior tendon thickness.

\section{Hyperosmolar dextrose injection}

A $2 \mathrm{ml}$ syringe was filled with $1 \mathrm{ml} 50 \%$ dextrose and $1 \mathrm{ml} 0.5 \%$ Marcaine to produce a $25 \%$ dextrose solution. The syringe was fitted to a connecting tube with a 25-gauge orange needle. All air was expelled before injecting and each treatment was performed under aseptic conditions. Injections were given to abnormal hypoechoic areas under US guidance with a $13 \mathrm{MHz}$ transducer (Fig. 4). The precise volume injected depended on the size of the tear and degree of resistance. 0.2-0.5 $\mathrm{ml}$ was injected into hypoechoic sites to a maximum of $1.5 \mathrm{ml}$. The tendon was then re-imaged to assess for injectate spread.

\section{Follow-up care}

Immediately after the injection the foot and ankle were immobilised in a walking boot (Aircast AirSelect Elite, DJO Global, USA) with heel raises to maintain plantar flexion and prevent tendon elongation. This was worn constantly during the 4-6 week immobilisation phase except for brief removal to wash, in a nonweight bearing position. Two day relative rest was ad-

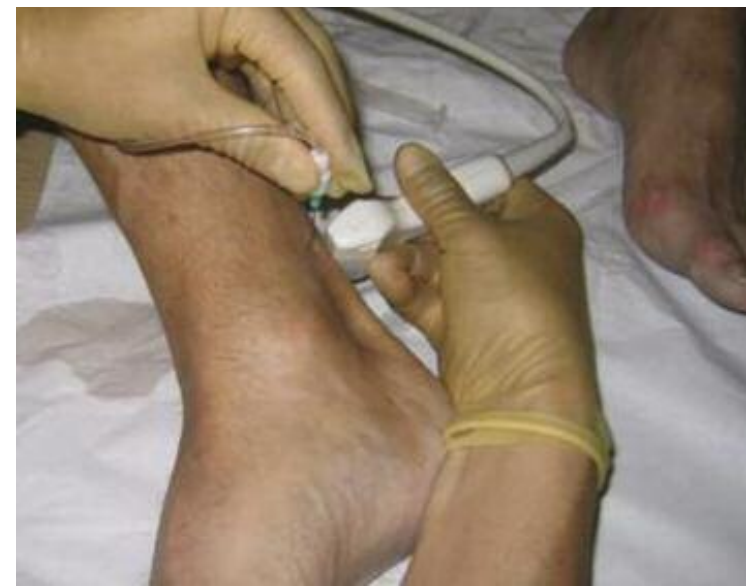

Figure 4. Ultrasound-guided dextrose injection procedure ${ }^{41}$.

vised and then patients were allowed to undertake low tendon-loading exercise such as static cycling with the boot strapped to the pedal of a static bicycle. Initially two heel raises were in situ but these were gradually removed over the course of the immobilisation period until the patient had no heel raises in situ for at least one week.

Once the boot was removed progressive tendon loading was prescribed by a consultant physiotherapist. In contrast to eccentric loading for tendinopathy, patients were instructed to stop if exercises provoked pain. The first two weeks focused on developing en- 
durance using isometric and concentric-eccentric exercises. Weight bearing calf raises (4 sets of 12) were carried out twice daily, taking 3 seconds per movement phase. Patients were able to exercise on a static bike at a high cadence ensuring they remained seated. After 2 weeks, load was introduced (up to $50 \%$ bodyweight) and the exercises were gradually progressed up to single leg calf raises performed over the edge of a step aiming to develop strength with the addition of load. Exercise on the static bike continued but incorporated some intervals off the seat. The later stages of rehabilitation (6 weeks onwards) focused on power and impact, such as enhancing performance using a limited stretch-shortening cycle approach. Running was introduced alongside sport-specific exercises. The initial focus was on increasing distance before developing speed, progressing ultimately to sprint starts in track spikes or equivalent. Rehabilitation progress was guided by pain, co-morbidities and patient goals.

\section{Data analysis}

Descriptive and mean comparisons were made using SPSS (version 20, IBM, UK). One-way Analysis of Variance (ANOVA) with a post hoc Tukey test was used to compare VISA-A scores and US characteristics before and after treatment. Spearman correlations were used to evaluate the relationship that sonographic variables and the volume injected had with VISA-A scores. A Jonckheere-Terpstra test was used to evaluate the trend between echogenicity grade and relative grayscale values. Statistical significance was set at a p-value of 0.05 .

\section{Results}

A total of 43 patients was sent the questionnaire and 30 responded (response rate $70 \%$ ). Participants had a mean age of 42 (SD 11.11). 63\% were males and $40 \%$ were elite athletes (Tab. I). The remaining par-

Table I. Patient data and questionnaire feedback.

\begin{tabular}{|c|c|c|c|c|c|c|c|c|}
\hline Age & Sex & $\begin{array}{l}\text { Side } \\
\text { Injured }\end{array}$ & Sport Level & $\begin{array}{l}\text { Baseline } \\
\text { VISA-A }\end{array}$ & $\begin{array}{l}3 \text { month } \\
\text { VISA-A }\end{array}$ & $\begin{array}{l}\text { Follow up } \\
\text { VISA-A } \\
\text { (months) }\end{array}$ & $\begin{array}{l}\text { Training/ } \\
\text { Competing? }^{\mathrm{a}}\end{array}$ & Satisfaction \\
\hline 37 & $\mathrm{~F}$ & $\mathrm{R}$ & Recreational & 19 & 69 & $79(3)$ & Modified training & Very \\
\hline 43 & M & $\mathrm{R}$ & Recreational & 61 & $20^{b}$ & $32(3)^{b}$ & Modified training & Not \\
\hline 27 & M & $\mathrm{L}$ & Elite & 53 & 83 & $84(7)$ & Not at previous level & Very \\
\hline 42 & M & $\mathrm{R}$ & Recreational & 62 & 62 & $68(5)^{\mathrm{c}}$ & Not at previous level & Moderate \\
\hline 52 & $\mathrm{~F}$ & $\mathrm{R}$ & Recreational & 34 & 88 & $91(6)$ & Modified training & Very \\
\hline 48 & $\mathrm{~F}$ & $\mathrm{~L}$ & Recreational & 20 & 33 & $80(8)^{\mathrm{e}}$ & Modified training & Not \\
\hline 43 & $\mathrm{~F}$ & L & Elite & 48 & 39 & $39(7)^{\mathrm{c}}$ & Modified training & Moderately \\
\hline 54 & $\mathrm{~F}$ & L & Recreational & 58 & 90 & $78(6)$ & Modified training & Very \\
\hline 38 & M & $\mathrm{R}$ & Recreational & 24 & 80 & $59(7)$ & Not at previous level & Very \\
\hline 52 & M & $\mathrm{L}$ & Elite & 28 & 75 & $75(12)$ & At previous level & Extremely \\
\hline 35 & $\mathrm{~F}$ & $\mathrm{R}$ & Recreational & 46 & 93 & $100(9)$ & Modified training & Extremely \\
\hline 64 & M & $\mathrm{R}$ & Elite & 13 & 71 & $71(3)$ & Modified training & Extremely \\
\hline 56 & M & $\mathrm{R}$ & Recreational & 29 & 95 & $95(12)$ & At previous level & Very \\
\hline 31 & $\mathrm{~F}$ & L & Elite & 0 & 88 & $100(10)$ & Modified training & Extremely \\
\hline 38 & $\mathrm{~F}$ & $\mathrm{~L}$ & Recreational & 42 & 34 & $21(12)^{\mathrm{de}}$ & None & Slightly \\
\hline 57 & M & $\mathrm{L}$ & None & 2 & 95 & $97(11)$ & Not at previous level & Extremely \\
\hline 57 & $M$ & $\mathrm{~L}$ & Recreational & 41 & 97 & $97(11)$ & Not at previous level & Very \\
\hline 50 & M & L & Recreational & 43 & 83 & $83(15)$ & Not at previous level & Moderately \\
\hline
\end{tabular}

to be continued 
Continue from Table I

\begin{tabular}{lllllllll}
\hline 33 & $\mathrm{M}$ & $\mathrm{L}$ & Elite & 29 & 60 & $63(19)$ & At previous level & Very \\
\hline 31 & $\mathrm{~F}$ & $\mathrm{R}$ & Recreational & 34 & 54 & $62(10)$ & Modified training & Very \\
\hline 42 & $\mathrm{M}$ & $\mathrm{L}$ & Recreational & 70 & 93 & $95(14)^{\mathrm{e}}$ & Not at previous level & Moderate \\
\hline 26 & $\mathrm{M}$ & $\mathrm{L}$ & Elite & 27 & 35 & $55(16)^{\mathrm{c}}$ & Not at previous level & Moderate \\
\hline 32 & $\mathrm{M}$ & $\mathrm{L}$ & Elite & 31 & 74 & $80(19)$ & Not at previous level & Extremely \\
\hline 31 & $\mathrm{M}$ & $\mathrm{L}$ & Elite & 40 & 73 & $100(12)$ & Modified training & Extremely \\
\hline 41 & $\mathrm{M}$ & $\mathrm{R}$ & Recreational & 36 & 66 & $67(19)$ & Not at previous level & Very \\
\hline 28 & $\mathrm{M}$ & $\mathrm{L}$ & Elite & 45 & 26 & $94(20)^{\mathrm{c}}$ & Modified training & Not \\
\hline 22 & $\mathrm{~F}$ & $\mathrm{~L}$ & Elite & 35 & 89 & $100(24)$ & Not at previous level & Extremely \\
\hline 33 & $\mathrm{~F}$ & $\mathrm{R}$ & Elite & 94 & 97 & $100(25)$ & Not at previous level & Very \\
\hline 53 & $\mathrm{M}$ & $\mathrm{R}$ & Recreational & 23 & 31 & $76(26)$ & Not at previous level & Very \\
\hline 51 & $\mathrm{M}$ & $\mathrm{L}$ & Recreational & 21 & 31 & $67(27)$ & Modified training & Moderately \\
\hline
\end{tabular}

a Level of sport/exercise after 3 months. b Suffered a recurrence of DVT two weeks after treatment. ${ }^{c}$ Received surgical treatment after 3-month follow-up. d Fell pregnant after 3-month follow-up. e Received High Volume Image Guided Injection (HVIGI) after 3-month-follow-up.

ticipants played recreationally bar one participant who did not play sport. The left side was marginally more commonly affected than the right (17:13). 57\% of participants had been previously diagnosed with Achilles tendinopathy but none of the participants had received treatment for the ITT or tendinopathy in the preceding 6 weeks.

$63 \%$ of patients was able to remember the precise moment that the tear occurred, the majority of which happened while sprinting. $90 \%$ could localize their pain with one finger.

\section{VISA-A scores}

Treatment increased VISA-A scores by an average of 31 (SD 30.54) points $(p<0.05)$ after 3 months, an $82 \%$ increase from baseline (Fig. 5). The majority of partici-

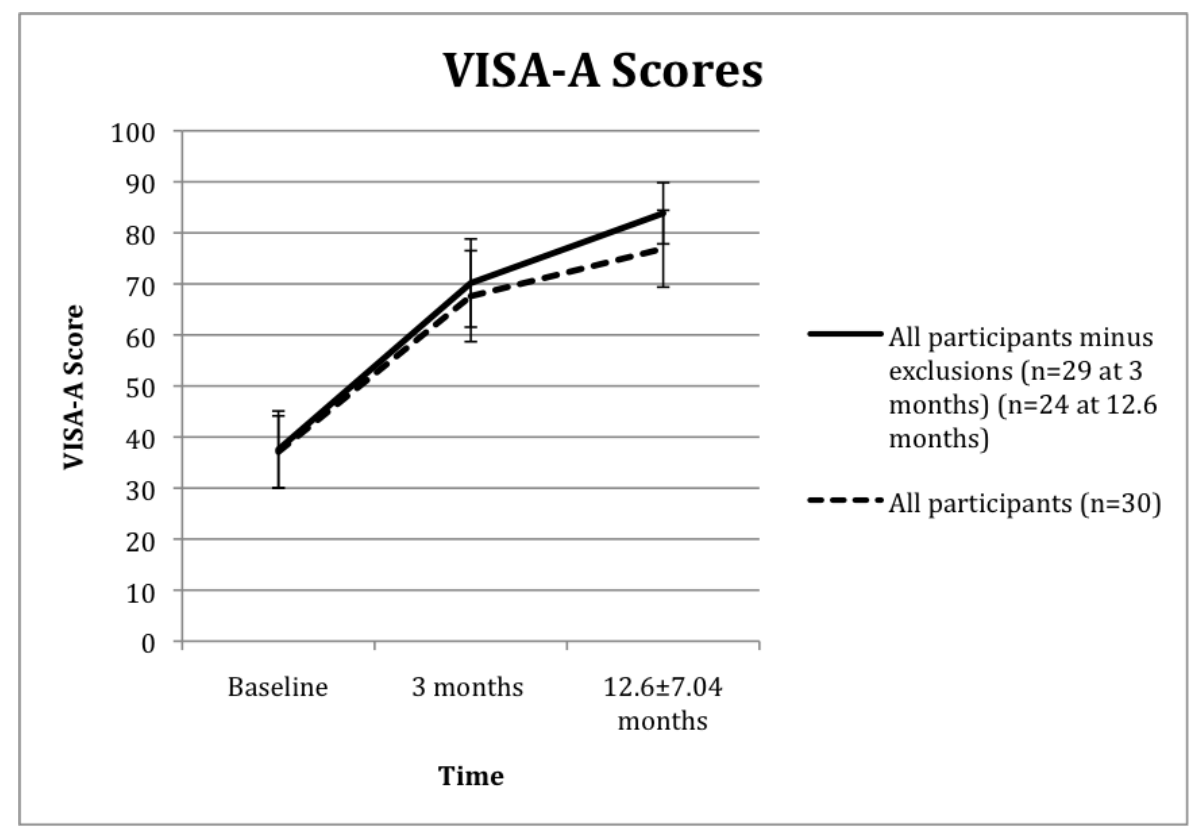

Figure 5. Mean VISA-A improvement $(95 \% \mathrm{Cl})$ following prolotherapy treatment. 
pants continued to improve and VISA-A scores had increased by 40 (SD 29.30) points at long-term follow-up $(p<0.05)$, a $107 \%$ increase from baseline. Although $97 \%$ of participants was playing sport after 3 months only $10 \%$ was competing at their previous level. This Figure had increased to $37 \%$ at long-term follow-up. One patient (with a history of previous DVTs) suffered a DVT two weeks after the injection. The boot had to be removed preventing him from completing his rehabilitation. No other adverse events occurred during the 3-month follow-up but 4 patients underwent surgery before the 12.6 (SD 7.04) month follow-up. Two had surgical debridement, one had a plantaris resection for ipsilateral Achilles tendinopathy and one had cheilectomy for ipsilateral Hallux Rigidius. One other patient fell pregnant between the 3-month and 12.6 (SD 7.04) month followup so did not complete the treatment. The VISA-A scores from these 6 patients were still included. Figure 5 illustrates the effect of excluding the DVT patient after baseline assessment and excluding the pregnant and 4 surgical patients after the 3-month follow-up.

\section{Sonographic evaluation}

All 30 patients were scanned before treatment and were scheduled a follow-up scan after treatment to assess sonographic variables (Tab. II). Four patients were unable to attend this appointment. One patient had left the country and one chose not to return, as they were not satisfied with treatment. The final two missed the follow- up appointment and did not respond to further contact. A total of 26 patients were scanned 5.2 (SD 2.29) weeks after treatment. In 7 patients the tear was no longer visible which corresponds to an echotexture grading of 0 and a relative grayscale value of 1 . There was no significant change in the size of the remaining tears, tendon thickness or the grade of neovascularisation. However, both measurement methods found improved echogenicity. The relative grayscale value was on average $31 \%$ higher at follow-up and the mean echogenicity grade had fallen by $55 \%(p<0.05)$. The Jonckheere-Terpstra test for ordered alternatives found a significant trend between echogenicity grade and relative grayscale values $(p<0.05)$.

The VISA-A score achieved after 3 months did not bear a significant correlation with the grade of neovascularisation $(r=-0.171, p>0.05)$ or grade of echogenicity ( $r=-$ $0.297, p>0.05$ ).

The neovessels observed with an ITT are quite different to those seen in Achilles tendinopathy. ITT patients often display much more nodular vessels when compared to the characteristic twig-like projections in tendinopathy patients (Fig. 6). The significance of this is not yet clear but could be a potential area for future research.

\section{Discussion}

\section{Principal findings}

This study has found that dextrose prolotherapy with immobilisation and rehabilitation can significantly im-

Table II. Sonographic variables.

\begin{tabular}{|c|c|c|c|}
\hline & Before treatment $^{a}$ & After immobilization & Mean change \\
\hline Tears with defined borders & $30 / 30$ & $19 / 26$ & \\
\hline Tear length (mm) & $17.61 \pm 13.89$ & $12.75 \pm 12.36$ & $\begin{array}{l}-28 \% \\
(p>0.05)\end{array}$ \\
\hline Tear width $(\mathrm{mm})$ & $5.42 \pm 3.25$ & $4.46 \pm 4.51$ & $-18 \%(p>0.05)$ \\
\hline Tendon thickness $(\mathrm{mm})$ & $7.94 \pm 1.91$ & $7.95 \pm 2.02$ & $\begin{array}{l}+0.13 \% \\
(p>0.05)\end{array}$ \\
\hline Neovascularisation grade $(0-5)^{\mathrm{b}}$ & $2.43 \pm 1.48$ & $2.31 \pm 1.54$ & $-4.9 \%(p>0.05)$ \\
\hline Echogenicity grade $^{c}$ & $2.07 \pm 0.58$ & $0.93 \pm 0.69$ & $-55 \%(p<0.05)$ \\
\hline Relative Grayscale value $^{d}$ & $0.61 \pm 0.09$ & $0.80 \pm 0.17$ & $+31 \%(p<0.05)$ \\
\hline
\end{tabular}

\footnotetext{
a Values are mean \pm standard deviation. ${ }^{b}$ Neovascularisation was graded on a modified Ohberg scale. This progresses from 0 (representing no Doppler signal) up to 5 (90\% of pathological area contains Doppler signal). ${ }^{c}$ Echogenicity was graded on a 0-3 ordinal scale. 0 represents normal echotexture. 1 represents a weakened echo signal with mild collagen fibre irregularity. 2 represents significant hypoechoic regions and fibre irregularity. 3 represents marked hypoechogenicity with anechoic regions. d Mean Grayscale values were calculated using ImageJ software. Darker, hypoechoic areas are represented by lower grayscale values. Homogenous tissue represented by a value of 1 .
} 


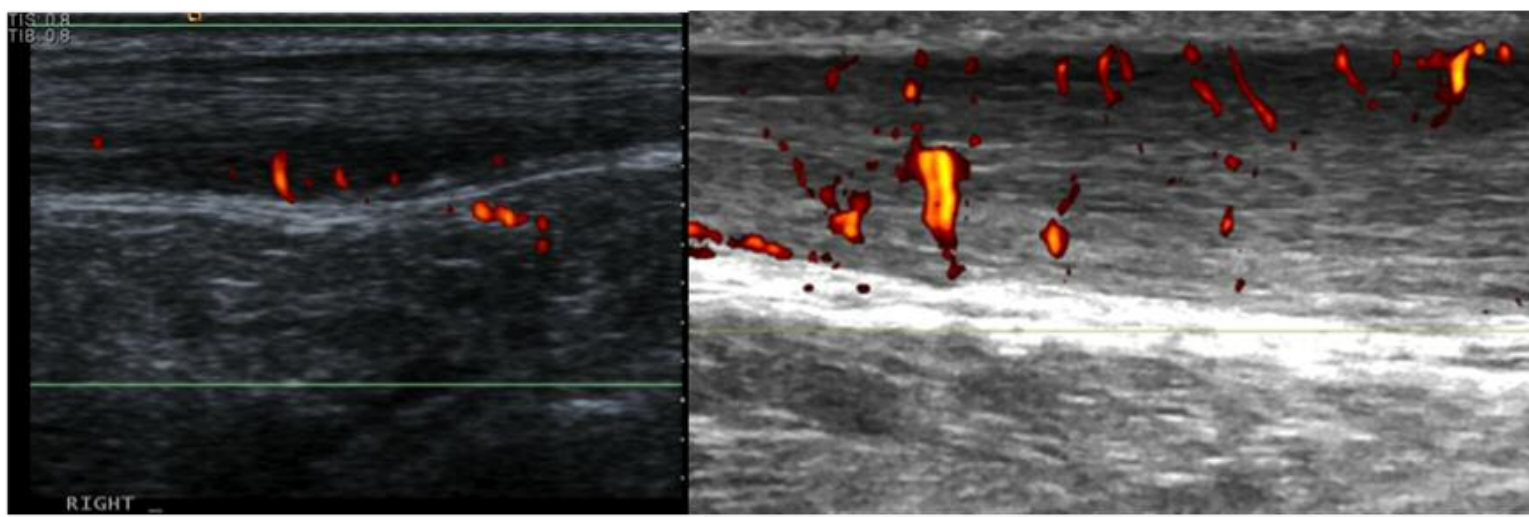

Figure 6. Neovascularisation of an ITT (left) in comparison to neovascularisation in midtendon Achilles tendinopathy (right).

prove pain and functional outcomes, measured with the VISA-A questionnaire. On US follow-up the tears were often filled in with echogenic material and the tear borders hard to distinguish. Tears that were still recognisable at follow-up had reduced echogenicity but the remaining characteristics were not significantly affected (Tab. II). This treatment package has been shown to be an effective intervention for intra-tendinous tears, albeit in an uncontrolled case series. These findings will help guide the clinical decisionmaking process when presented with acute, localised Achilles pain.

\section{Strengths and weaknesses}

The lack of knowledge on ITTs presents a number of limitations. Although the VISA-A is a very good assessment tool that allows comparisons with other studies investigating different Achilles pathologies, an ITT specific tool would allow more accurate outcome assessment. A larger sample size would also improve precision of results but all of these problems should be overcome as recognition and understanding of this pathology grows.

The study design also presents limitations. The retrospective nature required some participants to remember symptoms from 2 years previously, which could easily induce recall bias. It is a case series and inclusion of a control group with randomisation would improve treatment assessment. The follow-up US evaluation typically occurred 5.2 weeks after treatment. This allowed us to evaluate the induction of treatment but was often too soon to observe structural tendon changes and the study would have benefited from a long-term sonographic follow-up. Previous studies have utilized MRI to assess tendon damage and this study could have incorporated both MRI and US with a view to evaluating which imaging modality bears closer correlation to the clinical picture ${ }^{20}$. Level of sport was either none, recreational or elite. The recreational category was predictably the largest and patients within this category may have vastly different exercise regimes. Specifying hours of sport/exercise each week could have improved accuracy in this area.

Prolotherapy has been used for a variety of musculoskeletal conditions but there is still some uncertainty surrounding its rationale $23-25$. Dextrose injections are thought to induce an inflammatory reaction by dehydrating cells ${ }^{14}$. This change in osmotic pressure leads to the release of inflammatory cytokines and chemotactic factors, which attracts granulocytes and macrophages 25,26 . These macrophages secrete growth factors that activate fibroblasts. This leads to increased collagen synthesis that strengthens connective tissue, reducing pain and disability, which in turn leads to improved functionality 27,28 .

ITT's do not have a specific measure for outcome assessment. Although the VISA-A questionnaire is specific to Achilles tendinopathy it was felt to be a more informative outcome measure than a VAS pain scale as it incorporates the domains of pain, function in daily living and sporting activity ${ }^{16}$. Mean VISA-A change after 3 months was 31 (SD 30.54) points despite the foot being immobilised for approximately half this period. A minimum clinically important change ( $\mathrm{MCIC})$ has not yet been set but previous studies have proposed values between 10 and 25 , suggesting that this study demonstrates a very good clinical improvement ${ }^{18-21,29,30}$. Return to sport is an essential outcome measure in the athletic population and although only $10 \%$ were competing at their previous level after 3 months, this had increased to $37 \%$ at long-term follow-up (12.6 months). $89 \%$ of the elite athletes were competing at previous level by long-term follow-up and this may be due to greater motivation or perhaps demonstrates the effectiveness of a more structured rehabilitation program ${ }^{31}$

\section{Relation to other studies}

Ryan et al. demonstrated that prolotherapy reduces the size of intra-tendinous tears observed on US however this study found no significant change ${ }^{10}$ ITTs often appear filled in with echogenic material on US assessment after prolotherapy treatment and the $31 \%$ increase in relative grayscale value supports this observation. This homogenization of tendon tissue 
may be consistent with the healing process that prolotherapy aims to stimulate.

There is mixed evidence regarding the correlation between degree of neovascularisation and severity of Achilles tendinopathy symptoms ${ }^{32-34}$. This relationship does not appear to exist when considering ITT's and a reduction in neovascularisation was not associated with a significant change in VISA-A scores. Mean echogenicity grade was significantly reduced from 2.06 to 0.93 after 3 months and although this appeared to bear more of a relationship with clinical outcomes, there was no significant correlation ( $r=-$ $0.30, p>0.05$ ).

\section{Meaning of the study}

The continual improvement in VISA-A scores after 3 months (Fig. 5) highlights the importance of the rehabilitation phase. Comparable injection treatments such as sclerotherapy and high volume injections often produce significant short-term reductions in pain but often rely on repeated injections to sustain these benefits $^{35-38}$. Some studies suggest that these treatments focus more on pain fibre disruption whereas prolotherapy aims to stimulate a tissue healing response 39,40 . Rehabilitation based on progressive loading is essential to develop this repair process and progress clinical improvement further.

Five patients did not experience clinical improvement. The change in VISA-A after 3 months ranged from a 93-point increase to a 41-point decrease. Identifying the factors responsible for this wide range is essential to determine the suitability of this treatment for particular patients. Interestingly, the only patient who did not play any sport achieved the largest VISA-A increase. The 5 patients that did not improve after 3 months had a significantly higher baseline VISA-A (51.6) than those recording improvement (34.2) $(p<0.05)$. A VISA-A score of 51.6 correlates with considerable functional impairment but these findings suggest that prolotherapy produces superior outcomes in patients with more severe disability. Patients with a baseline score below 40 recorded a mean 43-point increase after 3 months whereas those with a baseline score above 40 achieved 33\% of this with a mean 14-point increase $(p<0.05)$. Conservative measures may be more appropriate to patients with less severe impairment, perhaps progressing to prolotherapy if symptoms deteriorate.

Figure 5 illustrates how patients that completed rehabilitation and did not seek additional treatments recorded greater improvement. Although 5 participants did not achieve an improved score, three of them still reported treatment satisfaction. These patients had received surgery or fallen pregnant during follow-up, which limited their activity considerably and lowered their VISA-A score. One patient was not satisfied with treatment despite recording clinical improvement at both follow-ups. This patient had concurrent tendinopathy and received an HVIGI after the 3-month follow-up. Her change in VISA-A score be- tween 3 months and 8 months was 3.6 times greater than the initial change from baseline to 3 months. ITTs and Achilles tendinopathy frequently co-exist ${ }^{6,10}$. This case highlights the importance of clinical assessment over radiological findings to determine which pathology is the primary driver of symptoms. Although, two other patients received HVIGls after the 3-month follow-up they did not elicit significant clinical improvement. Their VISA-A scores were $2 \%$ higher and $38 \%$ lower after 14 months and 12 months respectively. The DVT patient experienced a considerable VISA-A reduction 3 months after the injection and future studies could incorporate a history of DVTs as an exclusion criterion because the immobilisation period in the boot is an integral part of the treatment package.

Dextrose injections by themselves are typically somewhat painful and an equal amount of Marcaine is added to reduce this pain. Very small volumes are injected but little is known on the effect that volume has on outcomes. Previous prolotherapy studies on tendinopathy treatment have stated the maximum or approximate amount injected into patients, however this

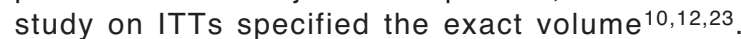
There was a weak inverse relationship between the volume injected and change in VISA-A scores after 3 months. Patients injected with less than $0.8 \mathrm{ml}$ achieved a $20 \%$ higher score than those injected with more than $0.8 \mathrm{ml}$. However, a significant correlation between volume injected and VISA-A change after 3 months did not exist $(r=0.134, p>0.05)$. Although, this study did not find a significant relationship, further investigation into the importance of volume may be needed to ensure the right patients are receiving the right amount.

\section{Future research}

This study has found that US guided dextrose injections followed by a period of immobilisation in an Aircast boot and a progressive rehabilitation program produced clinically significant improvements in patients with an intra-tendinous Achilles tear. However, randomized controlled trials are warranted to confirm these results and to improve future management of the intra-tendinous tear.

\section{Acknowledgements}

Many thanks go to the rehabilitation and imaging staff at the London Independent Hospital. We would also like to acknowledge our referral sources, without whom such large case series would not be available for pattern recognition, innovation and analysis.

\section{Conflict of interest}

No other relationships/conditions/circumstances that present potential conflict of interest. 


\section{References}

1. O'Brien M. The anatomy of the Achilles tendon. Foot Ankle Clin. 2005;10:225-238.

2. Sobhani S, Dekker R, Postema K, Dijkstra PU. Epidemiology of ankle and foot overuse injuries in sports: A systematic review. Scand J Med Sci. 2013;23:669-686.

3. Alfredson $\mathrm{H}$. Midportion Achilles tendinosis and the plantaris tendon. Br J Sports Med. 2011;45:1023-1025.

4. Scharfbillig RW, Jones S, Scutter S. Sever's disease: a prospective study of risk factors. J Am Podiatr Med Assoc. 2011;101:133-145.

5. Longo UG, Ronga M, Maffulli N. Achilles tendinopathy. Sports Med Arthrosc. 2009;17:112-126.

6. Chan O, Morton S, Pritchard M, Parkes T, Malliaras P, Maffuli N, Crisp T, Padhiar N, King J, Morrissey D. Intra-tendinous tears of the Achilles tendon - a new pathology? Analysis of a large 4 year cohort. MTLJ. [In review]

7. Cook JL, Khan KM, Kiss ZS, Purdam CR, Griffiths L. Reproducibility and clinical utility of tendon palpation to detect patellar tendinopathy in young basketball players. Victorian Institute of Sport tendon study group. Br J Sports Med. 2001;35:65-69.

8. Distel LM, Best TM. Prolotherapy: a clinical review of its role in treating chronic musculoskeletal pain. Am J Phys Med Rehabil. 2011;3:78-81.

9. Yoshii Y, Zhao C, Schmelzer JD, Low PA, An KN, Amadio PC. Effects of multiple injections of hypertonic dextrose in the rabbit carpal tunnel: a potential model of carpal tunnel syndrome development. Hand. 2014;9:52-57.

10. Ryan M, Wong A, Rabago D, Lee K, Taunton J. Ultrasoundguided injections of hyperosmolar dextrose for overuse patellar tendinopathy: a pilot study. Br J Sports Med. 2011;45:972977.

11. Ryan M, Wong A, Taunton J. Favorable outcomes after sonographically guided intratendinous injection of hyperosmolar dextrose for chronic insertional and midportion achilles tendinosis. AJR Am J Roentgenol. 2010;194:1047-1053.

12. Maxwell NJ, Ryan MB, Taunton JE, Gillies JH, Wong AD. Sonographically guided intra-tendinous injection of hyperosmolar dextrose to treat chronic tendinosis of the Achilles tendon: a pilot study. AJR Am J Roentgenol. 2007;189:W215220.

13. Sanderson LM, Bryant A. Effectiveness and safety of prolotherapy injections for management of lower limb tendinopathy and fasciopathy: a systematic review. Journal of Foot and Ankle Research. 2015;8:57.

14. Banks AR. A rationale for prolotherapy. J Orthop Med. 1991 13:54-59.

15. Padulo J, Oliva F, Frizziero A, Maffulli N. Muscles, Ligaments and Tendons Journal - Basic principles and recommendations in clinical and field science research: 2016 Update. MLTJ. 2016; 6(1):1-5

16. Robinson JM, Cook JL, Purdam C, Visentini PJ, Ross J, Maffulli N, Taunton JE, Khan KM. The VISA-A questionnaire: a valid and reliable index of the clinical severity of Achilles tendinopathy. Br J Sports Med. 2001;35:335-341.

17. Rahm S, Spross C, Gerber F, Farshad M, Buck FM, Espinosa $\mathrm{N}$. Operative treatment of chronic irreparable Achilles tendon ruptures with large flexor hallucis longus tendon transfers. Foot Ankle Int. 2013;34:1100-1110.

18. Bell KJ, Fulcher ML, Rowlands DS, Kerse N. Impact of autologous blood injections in treatment of mid-portion Achilles tendinopathy: double blind randomised controlled trial. Bmj. 2013;346:f2310

19. de Vos RJ, Weir A, van Schie HT, Bierma-Zeinstra SM, Verhaar JA, Weinans $\mathrm{H}$, Tol JL. Platelet-rich plasma injection for chronic Achilles tendinopathy: a randomized controlled trial. JAMA. 2010;303:144-149.
20. Khan KM, Forster BB, Robinson J, Cheong Y, Louis L, Maclean L, Taunton JE. Are ultrasound and magnetic resonance imaging of value in assessment of Achilles tendon disorders? A two year prospective study. Br J Sports Med. 2003:37:149-153.

21. Iversen JV, Bartels EM, Langberg $\mathrm{H}$. The victorian institute of sports assessment - achilles questionnaire (visa-a) - a reliable tool for measuring achilles tendinopathy. Int J Sports Phys Ther. 2012;7:76-84.

22. Malliaras P, Chan O, Simran G, Martinez de Albornoz P, Morrissey $\mathrm{D}$, Maffulli N. Doppler ultrasound signal in Achilles tendinopathy reduces immediately after activity. Int J Sports Med. 2012;33:480-484.

23. Dagenais S, Yelland MJ, Del Mar C, Schoene ML. Prolotherapy injections for chronic low-back pain. Cochrane Database Syst Rev. 2007;Cd004059.

24. Topol GA, Reeves KD, Hassanein KM. Efficacy of dextrose prolotherapy in elite male kicking-sport athletes with chronic groin pain. Arch Phys Med Rehabil. 2005;86:697-702.

25. Rabago D, Best TM, Zgierska AE, Zeisig E, Ryan M, Crane D. A systematic review of four injection therapies for lateral epicondylosis: prolotherapy, polidocanol, whole blood and platelet-rich plasma. Br J Sports Med. 2009;43:471-481.

26. Freeman JW, Empson YM, Ekwueme EC, Paynter DM, Brolinson PG. Effect of prolotherapy on cellular proliferation and collagen deposition in MC3T3-E1 and patellar tendon fibroblast populations. Transl Res. 2011;158:132-139.

27. Maynard JA, Pedrini VA, Pedrini-Mille A, Romanus B, Ohlerking $F$. Morphological and biochemical effects of sodium morrhuate on tendons. J Orthop Res. 1985;3:236-248.

28. Aneja A, Karas SG, Weinhold PS, Afshari HM, Dahners LE. Suture plication, thermal shrinkage, and sclerosing agents: effects on rat patellar tendon length and biomechanical strength. Am J Sports Med. 2005;33:1729-1734.

29. Tumilty S, Munn J, Abbott JH, McDonough S, Hurley DA, Baxter GD. Laser therapy in the treatment of achilles tendinopathy: a pilot study. Photomed Laser Surg. 2008;26:25-30.

30. Sayana MK, Maffulli N. Eccentric calf muscle training in nonathletic patients with Achilles tendinopathy. J Sci Med Sport. 2007;10:52-58.

31. Maffulli N, Testa V, Capasso G, Oliva F, Sullo A, Benazzo F, Regine R, King JB. Surgery for chronic Achilles tendinopathy yields worse results in nonathletic patients. Clin J Sport Med. 2006;16:123-128.

32. Leung JL, Griffith JF. Sonography of chronic Achilles tendinopathy: a case-control study. J Clin Ultrasound. 2008; 36:2732.

33. Finnoff JT, Fowler SP, Lai JK, Santrach PJ, Willis EA, Sayeed YA, Smith J. Treatment of chronic tendinopathy with ultrasound-guided needle tenotomy and platelet-rich plasma injection. Pm r. 2011;3:900-911.

34. De Jonge S, Warnaars JL, De Vos RJ, et al. Relationship between neovascularization and clinical severity in Achilles tendinopathy in 556 paired measurements. Scand J Med Sci Sports. 2013;24:773-778.

35. Maffulli N, Spiezia F, Longo UG, Denaro V, Maffulli GD. High volume image guided injections for the management of chronic tendinopathy of the main body of the Achilles tendon. Phys Ther Sport. 2013;14:163-167.

36. Alfredson $\mathrm{H}$, Ohberg L. Sclerosing injections to areas of neovascularisation reduce pain in chronic Achilles tendinopathy: a double-blind randomised controlled trial. Knee Surg Sports Traumatol Arthrosc. 2005;13:338-344.

37. Alfredson $\mathrm{H}$, Ohberg L, Zeisig E, Lorentzon R. Treatment of midportion Achilles tendinosis: similar clinical results with US and CD-guided surgery outside the tendon and sclerosing polidocanol injections. Knee Surg Sports Traumatol Arthrosc. 2007;15:1504-1509. 
38. Coombes BK, Bisset L, Vicenzino B. Efficacy and safety of corticosteroid injections and other injections for management of tendinopathy: a systematic review of randomised controlled trials. Lancet. 2010;376:1751-1767.

39. Chan O, O'Dowd D, Padhiar N, et al. High volume image guided injections in chronic Achilles tendinopathy. Disabil Rehabil.
2008:30:1697-1708.

40. Andres BM, Murrell GA. Treatment of tendinopathy: what works, what does not, and what is on the horizon. Clin Orthop Relat Res. 2008;466:1539-1554.

41. Chan O. Prolotherapy Injection. 2005. Photograph. London Independent Hospital, London. 